\title{
Caractérisation biologique de Schistosoma haematobium, $S$. bovis et leurs hybrides chez l'homme et chez les mollusques Bulinus truncatus naturellement infestés, au Centre et Nord de la Côte d'Ivoire.
}

\author{
Sonya C. GLITHO1*, Yves-Nathan T. TIAN-BI², Nana Rose DIAKITÉ1, Cyrille Koffi KONAN1, Eliézer \\ Kouakou N'GORAN 1,3. \\ ${ }^{1}$ Laboratoire de Biologie et Santé, UFR Biosciences, Université Félix Houphouët-Boigny, 22 B.P. 582, Abidjan 22, \\ Côte d'Ivoire. \\ 2 Laboratoire de Biotechnologie, Agriculture et Valorisation des Ressources Biologiques, UFR Biosciences, Université \\ Félix Houphouët-Boigny 22 B.P. 582, Abidjan 22, Côte d'Ivoire. \\ ${ }^{3}$ Centre Suisse de Recherches Scientifiques (CSRS) en Côte d'Ivoire, 01 BP 1303 Abidjan 01, Côte d'Ivoire.
}

Original submitted in on $7^{\text {th }}$ January 2021. Published online at www.m.elewa.org/journals/ on 28 th February 2021 https://doi.org/10.35759/JABs.158.8

\section{RÉSUMÉ}

Objectif : Identifier les espèces Schistosoma haematobium, S. bovis et leurs hybrides et, évaluer la compatibilité des schistosomes avec les mollusques hôtes intermédiaires et la souris blanche (Mus musculus albinos), hôte définitif, en infestation expérimentale.

Méthodologie et résultats : Des schistosomes ont été obtenus à partir de bulins infestés naturellement ou expérimentalement avec des miracidiums provenant des urines de l'homme. Ils ont permis d'étudier la compatibilité de quatre populations de Bulinus truncatus avec deux souches du groupe S. haematobium. La chronobiologie cercarienne a été étudiée à partir de quatre tranches horaires : 6-10h, 10-12h, 12-15h et 1518h. Des souris blanches infestées expérimentalement, ont été perfusées pour dénombrer les vers adultes. Une meilleure compatibilité schistosome-mollusque a été observée au niveau des infestations sympatriques. Le pic d'émergence cercarienne pour les mollusques naturellement infestés a été observé entre 6-10h, 10h$15 \mathrm{~h}$ et à 15-18h, tandis que celui des bulins infestés expérimentalement a été majoritairement observé entre 10-15h. En termes de nombre de vers collectés, les souris blanches ont été plus permissives aux schistosomes provenant des mollusques naturellement infestés.

Conclusion et application des résultats : Les pics d'émergence cercarienne précoce (6-10h) et tardif (15-18h) pourraient être liés à $S$. bovis, tandis que celui de 10-15h correspondrait à $S$. haematobium. De même, en raison de la très faible compatibilité connue entre Mus musculus albinos et $S$. haematobium, les schistosomes auxquels les souris blanches ont été plus permissives sont fort probablement des $S$. bovis et/ou hybrides $S$. bovis $\times$ S. haematobium. Ainsi, les espèces anthropophiles et celles zoophiles du groupe Schistosoma haematobium peuvent être distinguées de manière routinière par deux approches. La première, en disséquant des souris de laboratoire (Mus musculus albinos) 4 mois après leur mise au contact de l'eau contenant des cercaires obtenues après exposition à la lumière de mollusques infestés prélevés sur le terrain ; la seconde en comparant les profils obtenus après dénombrement des cercaires émises au cours de quatre tranches horaires bien choisies.

Mots clés : Bulinus truncatus, Schistosoma haematobium, Schistosoma bovis, Caractérisation, Chronobiologie. 


\section{ABSTRACT}

Objective: Identify the species Schistosoma haematobium, S. bovis and their hybrids; to evaluate the compatibility of schistosomes with intermediate host snails and the white mouse (Mus musculus albinos), the definitive host for experimental infestation.

Methodology and results: Schistosomes have been obtained from naturally or experimentally infested snails with miracidiums from human urine. The compatibility of four populations of Bulinus truncatus with two strains of the S. haematobium group was studied. The chronobiology of cercariae was studied in four time slots: 610h, 10-12h, 12-15h and 15-18h. Experimentally infested white mice were perfused and adult worms were collected. A better schistosome-snail compatibility was observed in sympatric infestations. The peak of cercarial emergence for naturally infested snails was at 6-10h, 10h-15h and 15-18h, while that of the snails experimentally infested was mostly observed at 10-15h. In terms of number of worms collected, white mice were more permissive to schistosomes from naturally infested snails.

Conclusion and application of results: Early (6-10h) and late (15-18h) cercarial emergence peaks can be related to $S$. bovis, while the $10-15 \mathrm{~h}$ peak correspond to $S$. haematobium. Due to the reported low compatibility between the white mice Mus musculus albinos and $S$. haematobium, the observed schistosomes permissive to the white mice are most likely S. bovis and/or hybrids S. bovis $\times$ S. haematobium. Therefore, anthropophilic and zoophilic species of the Schistosoma haematobium group can be routinely distinguished by two approaches. The first, dissecting laboratory mice (Mus musculus albinos) 4 months after their contact with water containing cercariae obtained after exposure to light from infested snails collected in the field; the second by comparing the profiles obtained after enumeration of cercariae emitted during four well-chosen time slots.

Keywords: Bulinus truncatus, Schistosoma haematobium, Schistosoma bovis, hybrid, Characterization, Chronobiology.

\section{INTRODUCTION}

Les schistosomiases constituent un problème de santé publique dans les pays en développement des régions tropicales et subtropicales (OMS, 2019). Elles sont dues principalement à six espèces parasitaires du genre Schistosoma (Gryseels et al., 2006). Le cycle de vie de ces schistosomes fait intervenir un mollusque d'eau douce, l'hôte intermédiaire, et un vertébré, l'hôte définitif. Les œufs de schistosomes éliminés dans les urines et les selles, éclosent au contact de l'eau douce pour libérer des larves ciliées, les miracidiums. Ces derniers infestent des mollusques hôtes intermédiaires compatibles et se transforment en cercaires par reproduction asexuée, au bout d'un mois environ. Ces cercaires émergent activement des mollusques infestés et nagent à la recherche d'un hôte définitif (l'homme ou l'animal vertébré) chez lequel elles pénètrent à travers la peau. Chez cet hôte, les cercaires deviennent des vers adultes mâles et femelles qui s'accouplent pour se reproduire. Les œufs pondus par la femelle sont excrétés à l'extérieur et le cycle peut recommencer
(Dorsey et al., 2002). En Côte d'lvoire, les schistosomiases sont endémiques sous les formes urogénitales due à Schistosoma haematobium et intestinale due à $S$. mansoni. Les deux mollusques hôtes intermédiaires de $S$. haematobium sont Bulinus truncatus et $B$. globosus, tandis que Biomphalaria pfeifferi est le mollusque hôte intermédiaire de S. mansoni (N'Guessan et al., 2015). Certaines espèces de schistosomes peuvent s'hybrider. Par exemple, des cas d'hybridation ont été rapportés d'une part, entre les schistosomes de l'homme, S. haematobium et S. mansoni (Boissier et al., 2016) ; et d'autre part, entre S. haematobium et l'agent de la schistosomiase du bétail $S$. bovis (Boissier et al., 2016 ; Tian-Bi et al., 2019 ; Webster et al., 2019 ; Angora et al., 2019). En Côte d'Ivoire, les outils moléculaires ont récemment permis de détecter des hybrides $S$. haematobium $x S$. bovis à partir de cercaires provenant des régions nord, centre (Tian-Bi et al., 2019), des miracidiums provenant du sud-est et ouest du pays (Angora et al., 2019). L'identification morphologique de ces 
parasites au niveau spécifique apparaît donc difficile. L'usage d'outils plus performants pour distinguer les schistosomes s'avère donc essentielle pour mieux comprendre l'épidémiologie des schistosomiases et mettre en place des stratégies efficaces de lutte et d'élimination (Huyse et al., 2013 ; N'Guessan et al., 2015 ; Leger et Webster, 2017). Les outils moléculaires sont non seulement onéreux, mais également difficiles d'usage en routine (Utzinger et al., 2015). II est alors nécessaire d'investiguer des alternatives plus

\section{MATÉRIEL ET MÉTHODES}

Sites et population d'étude : Cette étude a été menée entre Novembre 2017 et Novembre 2018, dans quatre localités de la Côte d'Ivoire situées au Centre (Kongobo et Linguebo) et au Nord-est (Djémitédouo et Korokara) (Figure 1). Le Centre et le Nord du pays sont caractérisés par un climat à deux saisons. La saison sèche s'étend de Novembre à Février au centre et d'Octobre à Mai au nord. La saison pluvieuse s'étend de accessibles. Les marqueurs biologiques tels que la compatibilité parasite-hôte et la chronobiologie cercarienne peuvent être envisagées comme approches pouvant aider à identifier les espèces de schistosomes. Toutefois, il est important d'en tester les performances dans les différents contextes. Cette étude vise ainsi à évaluer la compatibilité parasite-hôte-et la chronobiologie cercarienne comme outils de caractérisation et d'identification de schistosomes du groupe Schistosoma haematobium et leurs hybrides éventuels.

Mars à Octobre et de Juin à Septembre. Les quatre localités abritent chacune une école primaire, un lac de barrage aménagé sur les affluents des fleuves Comoé (Djémitédouo) et Bandama (Korokara, Kongobo et Linguebo). La schistosomiase urogénitale est présente dans ces localités (Tian Bi et al., 2018). La population d'étude est composée des enfants d'âge scolaire de 7 à 10 ans.

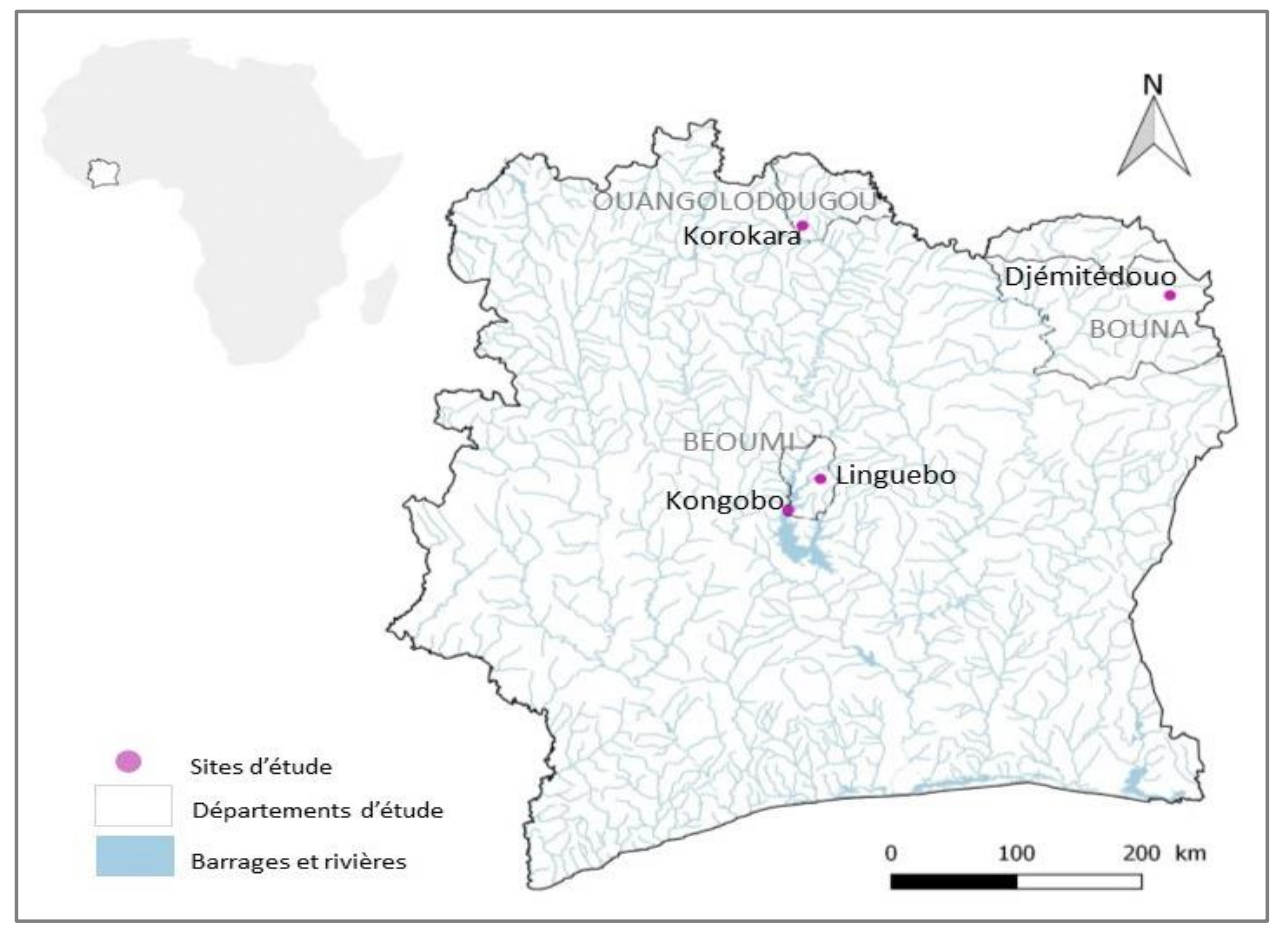

Figure 1 : Localisation des sites d'étude

Collecte des mollusques adultes et obtention des jeunes bulins : Les mollusques ont été collectés à l'aide d'une épuisette et d'une pince à bouts minces. La collecte à l'épuisette a consisté à faucher dans la végétation. L'observation des supports flottants a permis la collecte des mollusques à l'aide d'une pince. Les tests d'émergence réalisés sur les mollusques adultes du genre Bulinus truncatus ont permis d'isoler les mollusques naturellement infestés par des schistosomes des mollusques sains. Ces derniers ont servi à réaliser 
la chronobiologie cercarienne et les infestations de souris. Soixante mollusques adultes (G0) (3 lots de 20 mollusques) par site, n'ayant pas émis de cercaire de schistosome à l'issue de la période des tests, ont permis d'obtenir de jeunes mollusques G1. Les mollusques adultes ont été isolés et élevés individuellement dans des piluliers contenant $10 \mathrm{~mL}$ d'eau de source puis nourris avec des feuilles de laitue bouillie et des granulés pour poissons (Tian Bi et al., 2018). Deux ou trois bulins juvéniles mesurant entre 1,8 et $2 \mathrm{~mm}$ de hauteur ont été choisis au hasard par adulte, de sorte à obtenir 50 jeunes mollusques par localité. Enfin, ces jeunes mollusques ont été infestés par les œufs de schistosomes provenant de l'homme pour évaluer la susceptibilité, réaliser la chronobiologie cercarienne et infester les souris.

Collecte des œufs de schistosomes : Les œufs de schistosomes ont été collectés chez les enfants consentants, présentant une hématurie (N'Goran et al., 2001). Quinze enfants âgés de 07 à 10 ans ont été choisis de manière aléatoire dans les localités de Kongobo au Centre et Djémitédouo au Nord-est. La collecte des œufs de schistosomes a été effectuée à partir de la technique de filtration des urines (Plouvier et Colette, 1975). Au laboratoire, les œufs mis à éclore dans l'eau de source ont permis d'obtenir deux souches de miracidiums selon l'origine géographique (Kongobo et Djémitédouo). Ces miracidiums ont été utilisés pour infester des jeunes $B$. truncatus.

Infestation expérimentale et susceptibilité des mollusques: Au niveau de chaque localité, des infestations sympatriques (Souche de schistosomes et $B$. truncatus de la même zone) et allopatriques (Souche de schistosomes et $B$. truncatus de zones différentes) ont été réalisées selon le protocole décrit par Tian $\mathrm{Bi}$ et al. (2018). Au niveau de chacune des quatre localités (Djémitédouo et Korokara, Kongobo et Linguebo), 50 mollusques juvéniles ont été soumis à l'infestation. Chaque type d'infestation a été répliqué trois fois, soit par localité 300 mollusques, donnant à un total de 1200 mollusques exposés. Après l'infestation, les mollusques ont été remis en élevage, à température ambiante, par lots de cinq individus par pilulier selon leurs provenances et le type d'infestation. Les mollusques ont été individuellement testés pour l'émission cercarienne, 30 jours après infestation (N'Goran, 1998). La susceptibilité des mollusques à l'infestation a été estimée à partir des taux de survie à l'infestation (Nombre de mollusques survivants/ Nombre de mollusques exposés) et de réussite à l'infestation (TRI), qui correspond au nombre total de mollusques positifs rapporté au nombre de mollusques survivants 30 jours après l'infestation.

Chronobiologie cercarienne : Les bulins ayant émis des cercaires ont été utilisées pour le suivi de la chronobiologie cercarienne. Cette dernière a consisté à compter le nombre de cercaires émises par les mollusques infestés par tranche horaire. Pour ce faire, les mollusques ont été placés dans des piluliers individuels contenant $10 \mathrm{ml}$ d'eau de source. Ils ont été exposés à la lumière artificielle pendant quatre tranches horaires : 6-10 heures, 10-12 heures, 12-15 heures et 15-18 heures. Après chaque tranche horaire, l'eau de bain a été filtrée à l'aide de filtres Nytrel placés sur des porte-filtres ajustés sur une seringue. Le filtre a été ensuite déposé sur une lame porte-objet et recouvert d'une goutte de lugol. Le nombre de cercaires émises a été dénombré au microscope optique à l'objectif x40 (Cecchi et al., 2007). Pour chaque zone géographique, les taux de cercaires émises ont été calculés par tranche horaire (proportion du nombre de cercaires émises durant la tranche horaire considérée/nombre total de cercaires émises au cours des quatre tranches horaires).

Infestation des souris et collecte des vers adultes : Les cercaires collectées à partir de ces mollusques ont été également utilisées pour réaliser l'infestation de souris blanches Mus musculus albinos. L'infestation des rongeurs a été faite selon le protocole décrit par Tian $\mathrm{Bi}$ et al. (2018), à partir des cercaires émises par les bulins infestés naturellement ou au laboratoire. Au bout de 120 jours après l'infestation, les souris ont été anesthésiées puis sacrifiées. Les vers adultes ont été collectés, au niveau de la veine porte-hépatique et dénombrés après perfusion d'une solution contenant du citrate de sodium (anticoagulant) et du chlorure de sodium au niveau du ventricule gauche du cœur de l'animal anesthésié. La pression exercée lors de la perfusion a ainsi fait décoller les vers adultes de la paroi de la veine porte-hépatique. Les souris infestées ont présenté un foie granuleux et ceux indemnes un foie lisse. La susceptibilité des souris à l'infestation a été estimée à partir du taux de réussite à l'infestation qui correspond au nombre total de souris survivantes au bout de 120 jours après l'infestation, rapporté au nombre de souris positives. Le nombre de vers adultes collectés par souris a permis d'estimer la charge parasitaire par zone géographique.

Considérations éthiques : Le protocole de l'étude a été approuvé par le Comité National d'Éthique des Sciences de la Vie et de la Santé (Réf. 05519/MSHP/CNESVS-KP). Par ailleurs, un consentement éclairé par écrit du parent ou du tuteur légal de chaque 
participant a été obtenu suite à l'explication des procédures de l'étude. Tous les enfants positifs des localités d'étude ont été traités après notre passage.

Analyses des données : Les taux de survie et de réussite à l'infestation (TRI) ont été comparés suivant le type d'infestation (sympatrique/allopatrique), entre

\section{RÉSULTATS ET DISCUSSION}

Susceptibilité des mollusques à l'infestation par les schistosomes: Les résultats présentés dans le tableau 2 montrent que, aussi bien les taux de survie que les taux de réussite à l'infestation ont significativement varié entre les combinaisons allopatriques et sympatriques. Ainsi, avec la souche parasitaire provenant du centre, les taux de survie et d'infestation zones géographiques et sites d'étude. Le test de chi2 $\left(X^{2}\right)$ a été utilisé pour comparer les taux d'infestation chez l'hôte intermédiaire, l'hôte définitif et les pics d'émergence cercarienne, entre zones géographiques. Les analyses statistiques ont été effectuées avec le logiciel stata 9.0.

des mollusques ont respectivement varié de façon significative de $34 \%$ à $50 \%(p=0,022)$ et de $29 \%$ à 44 $\%(p=0.028)$ entre infestations allopatriques et sympatriques. Pour la souche parasitaire provenant du Nord ; ces taux ont respectivement varié de façon significative de $37 \%$ à $52 \%(p=0,033)$ pour la survie et de $27 \%$ à $42 \% ;(p=0,026)$ pour l'infestation. 
Glitho et al., J. Appl. Biosci. 2021 Caractérisation biologique de Schistosoma haematobium, S. bovis et leurs hybrides chez l'homme et chez les mollusques Bulinus truncatus naturellement infestés, au Centre et Nord de la Côte d'Ivoire.

Tableau 2 : Variation des taux de survie et de réussite à l'infestation des mollusques selon le type d'infestation

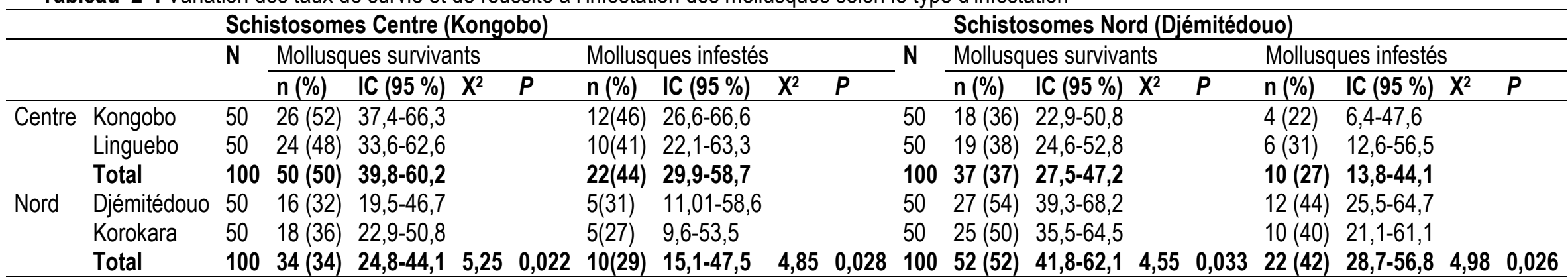

$\mathbf{N}$ : Mollusques exposés ; $\mathbf{n}$ : Mollusques survivants et infestés ; \% : Pourcentage ; $\boldsymbol{P}$ : Probabilité ; IC : Intervalle de confiance 
Chronobiologie cercarienne des mollusques naturellement infestés : Les pics d'émergence des cercaires des mollusques naturellement infestés ont présenté des profils distincts. Au centre, sur les neuf mollusques exposés, huit (08) ont émis plus de cercaires entre 10h-15h (88\%) et un (01) a eu un profil différent en plateau (12\%) (Figure 2). Au nord, sur les dix-huit (18) mollusques exposés, quinze (15) ont émis plus de cercaires entre $06 \mathrm{~h}-10 \mathrm{~h}, 15 \mathrm{~h}-18 \mathrm{hh}(83 \%)$ et deux (02) entre 10h-12h, 12h-15h (11\%); un mollusque (01) a eu un profil différent en plateau (6\%) (Figure 3).

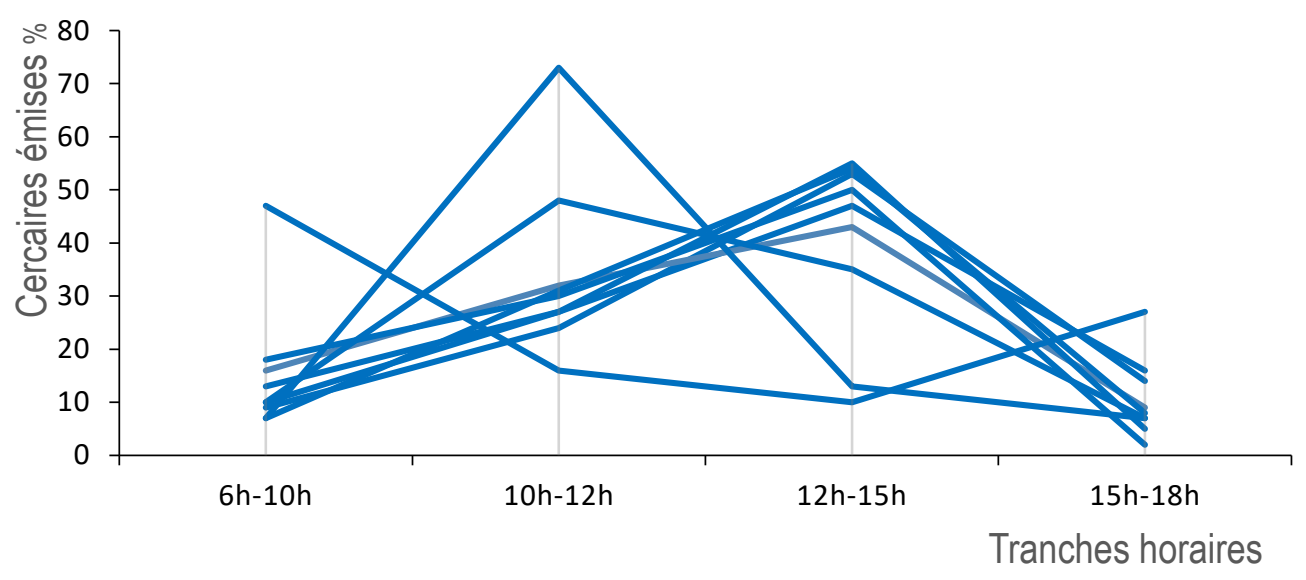

Figure 2: Variation de l'émission cercarienne par tranche horaire des mollusques naturellement infestés par les schistosomes du centre de la Côte d'Ivoire.

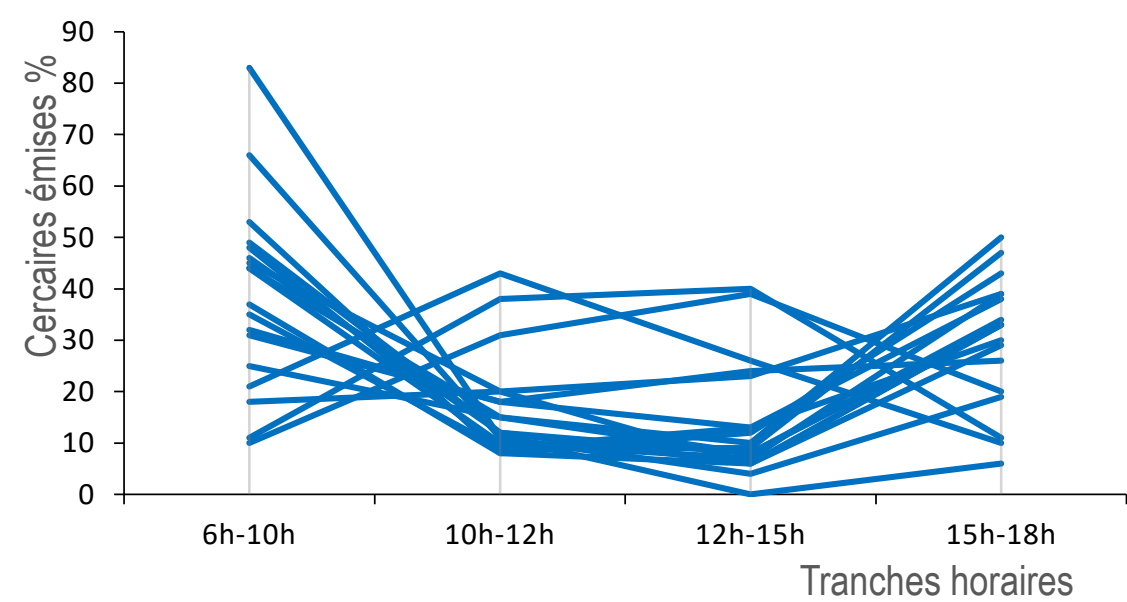

Figure 3 : Variation de l'émission cercarienne par tranche horaire des mollusques naturellement infestés par les schistosomes du nord de la Côte d'Ivoire.

Chronobiologie cercarienne des mollusques expérimentalement infestés : Les pics d'émergence des cercaires des mollusques expérimentalement infestés à partir des miracidiums provenant de l'homme ont présenté des profils distincts. Au centre, sur les dix (10) mollusques exposés, trois (03) ont émis plus de cercaires entre $10 \mathrm{~h}-12 \mathrm{~h}(30 \%)$ et cinq (05) ont émis plus de cercaires entre 12h-15h (50\%); deux (02) mollusques ont eu des profils différents en plateau ( 20 $\%$ ) (Figure 4). Au nord, sur les dix (10) mollusques exposés, cinq (05) ont émis plus de cercaires entre 10h$12 \mathrm{~h}(50 \%)$ et cinq (05) entre 12h-15h (50\%) (Figure 5). 


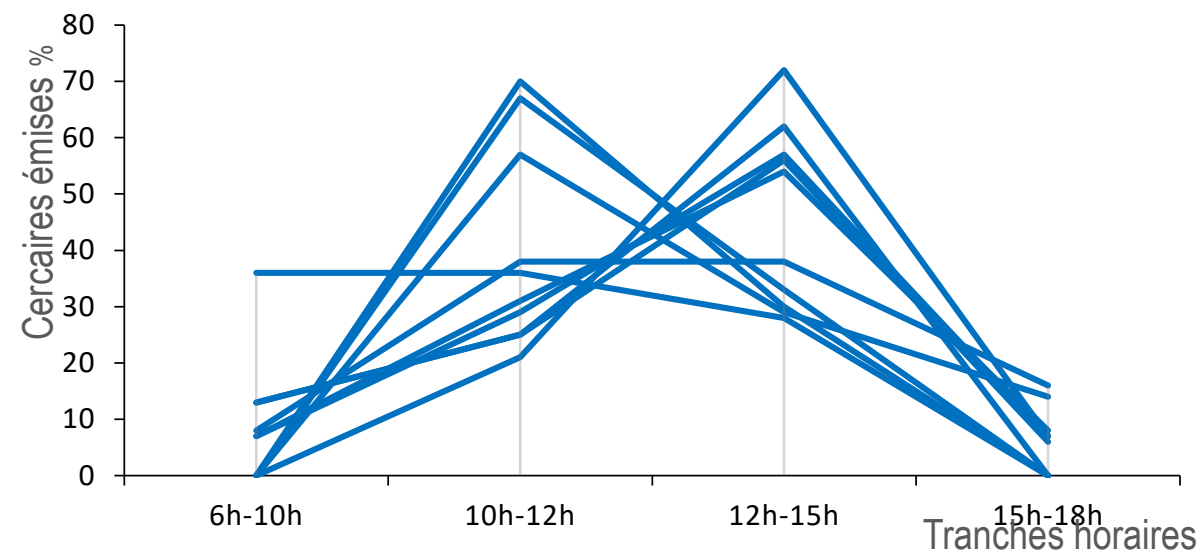

Figure 4 : Variation de l'émission cercarienne par tranche horaire des mollusques expérimentalement infestés par des schistosomes prélevés dans les urines des écoliers au centre de la Côte d'Ivoire.

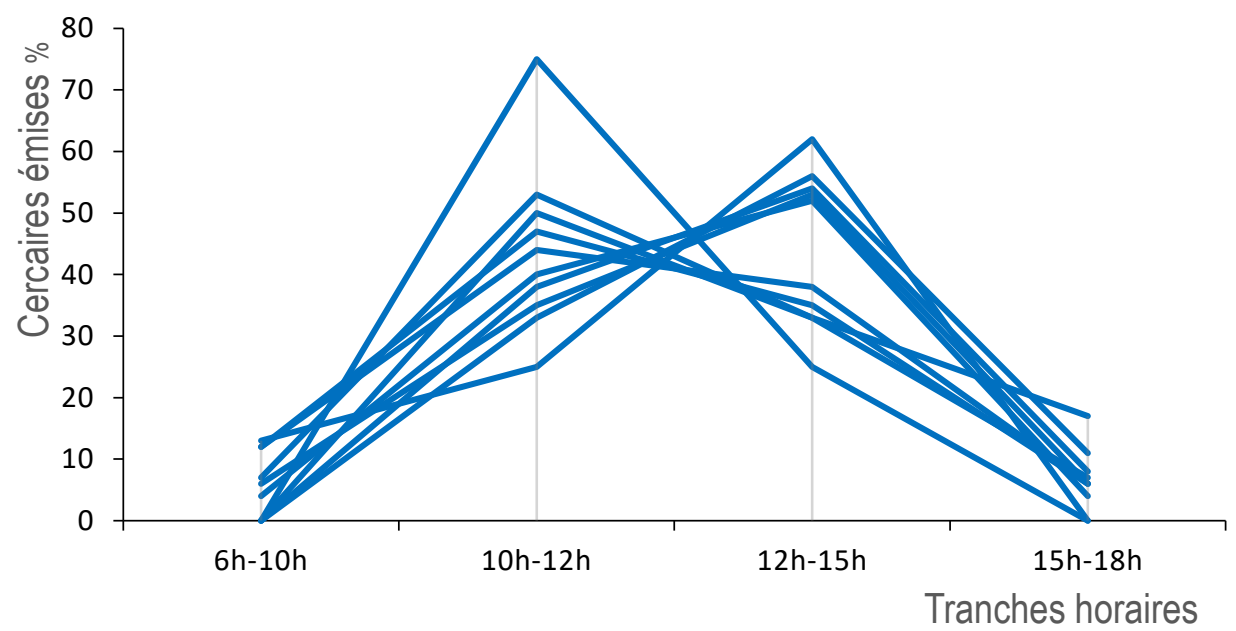

Figure 5 : Variation de l'émission cercarienne par tranche horaire des mollusques expérimentalement infestés par des schistosomes prélevés dans les urines des écoliers au nord de la Côte d'Ivoire

Charge parasitaire des souris infestées par des cercaires : Les perfusions des souris et l'observation visuelle des foies n'ont pas permis de mettre en évidence des schistosomes adultes chez les rongeurs exposés aux cercaires émises par les bulins infestés à partir des miracidiums provenant des urines humaines des participants du centre et du nord de la Côte d'Ivoire. Aucun ver adulte n'a ainsi été obtenu chez les souris exposées aux cercaires issues de mollusques infestés au laboratoire. Par contre des vers adultes ont été collectés au niveau des souris exposées à des cercaires issues de mollusques naturellement infestés provenant de ces mêmes zones. Le taux de réussite à l'infestation au centre a été inférieur à celui du nord $(6 \%$ vs $42 \% ; p$ $=0,0001$ ) (Tableau 3). La charge parasitaire chez les souris a été plus élevée au nord qu'au centre. En effet, sur un total de 251 vers adultes, 11 provenaient du centre (4\%) et 240 provenaient du nord (96\%). (4\% vs $96 \% ; p<0,0001$ ) (Tableau 4). 
Glitho et al., J. Appl. Biosci. 2021 Caractérisation biologique de Schistosoma haematobium, S. bovis et leurs hybrides chez l'homme et chez les mollusques Bulinus truncatus naturellement infestés, au Centre et Nord de la Côte d'Ivoire.

Tableau 3 : Taux de survie et de réussite à l'infestation chez les souris

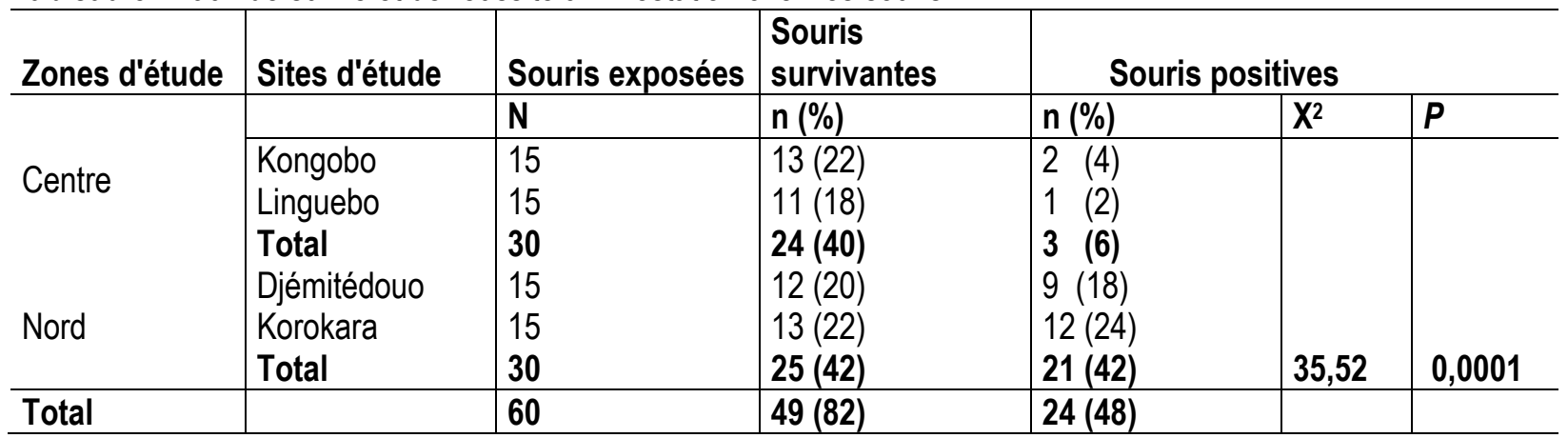

$\mathbf{N}$ : Souris exposées ; $\mathbf{n}$ : Souris survivantes et positives ; \% : Pourcentage ; $\boldsymbol{P}:$ Probabilité

Tableau 4 : Charge parasitaire des souris infestées par des cercaires collectées dans le milieu naturel

\begin{tabular}{ccccc}
\hline Zones d'étude & Sites d'étude & \multicolumn{3}{c}{ Vers collectés (N=251) } \\
\hline \multirow{4}{*}{ Centre } & & $\mathbf{n}(\%)$ & $\mathbf{X}^{2}$ & $P$ \\
& Kongobo & $7(3)$ & & \\
& Linguebo & $4(1)$ & & \\
\multirow{2}{*}{ Nord } & Total & $11(4)$ & & \\
& Djémitédouo & $77(31)$ & & \\
& Korokara & $163(65)$ & $\mathbf{5 3 . 3 3}$ & $\mathbf{0 , 0 0 0 1}$
\end{tabular}

$\mathbf{N}$ : Vers adultes collectés $; \mathbf{n}$ : Vers adultes collectés par site ; \% : Pourcentage ; $\boldsymbol{P}$ : Probabilité

\section{DISCUSSION}

Dans cette étude, il nous a été donné d'observer avec les différents marqueurs biologiques utilisés une variabilité des souches parasitaires de schistosomes des localités du Centre et du nord. Ainsi, il a été observé une différence significative entre les taux de survie et de réussite à l'infestation des mollusques lors des infestations sympatriques et allopatriques. Ces taux, traduisant la compatibilité mollusque-schistosome ont été plus élevés pour les infestations sympatriques que pour les infestations allopatriques. En effet, cela pourrait être l'expression d'une meilleure adaptation des parasites à leurs populations locales de bulins. Les gènes parasitaires responsables de l'infectiosité et ceux intervenant dans la défense au niveau de l'hôte coévolueraient pour assurer une interaction durable (Moné et al., 2011 ; Portet et al., 2019), induisant une meilleure adaptation du parasite à son hôte dans la combinaison sympatrique qu'allopatrique (Moné et al., 2011; N'Guessan et al. 2015). Le marqueur chronobiologie cercarienne a permis de distinguer, des profils types d'espèces de schistosomes anthropophiles, avec des pics d'émergences situés entre 10 heures et 15 heures, des espèces de schistosomes zoophiles, avec des pics d'émergences situés entre 6 heures et 10 heures, entre 15 heures et 18 heures. Ces tranches horaires correspondraient aux périodes où les cercaires auraient une probabilité élevée de rencontrer l'homme ou le bétail dans l'eau (N'Goran,1998 ; Mintsa et al., 2014 ; Boissier et al., 2016 ; M'Bra et al., 2018). A l'exception de deux mollusques du centre qui ont présenté des profils atypiques, tous les mollusques infestés expérimentalement à partir des miracidiums provenant des urines de l'homme tant du centre que du nord ont présenté des profils d'émergence de type anthropophile. Par contre, tous les mollusques naturellement infestés du centre ont présenté un profil anthropophile à l'exception d'un qui a présenté un profil zoophile. Ceux du nord ont quant à eux présenté majoritairement des profils zoophiles. II en ressort donc que l'espèce retrouvée chez l'homme au centre et au nord de la Côte d'Ivoire serait $S$. haematobium. Toutefois, les profils atypiques observés dont l'un a présenté une forte proportion de cercaires émises entre 6 heures et 10 heures interpellent sur la présence éventuelle des œufs d'hybrides dans les urines de l'homme. II est par ailleurs observé que $S$. haematobium et $S$. bovis sont présentes dans les deux zones considérées avec toutefois la première très majoritaire au centre quand la seconde domine au nord (N'Goran, 1998, Tian bi et al., 2019). L'infestation des souris blanches par les cercaires issues des mollusques expérimentalement infestés à partir des urines humaines n'a donné aucun ver adulte. Cela 
confirme le fait que la souris blanche soit un mauvais hôte pour l'espèce $S$. haematobium. II est en effet, reconnu que le mérion et le hamster sont des hôtes de choix de $S$. haematobium en conditions contrôlées. L'infestation des souris blanches avec les parasites issus des mollusques provenant du milieu naturel a donné des taux de réussite à l'infestation, plus élevés au nord qu'au centre du pays. De plus, les vers obtenus à partir des mollusques du centre étaient tous des mâles, alors qu'au nord des vers mâles et femelles ont été obtenus. Le faible taux de réussite à l'infestation et l'absence de vers femelles observés au centre pourraient s'expliquer par la prédominance dans cette zone, de S. haematobium, qui infeste difficilement la souris blanche (Cheever et al., 1983 ; Jacobs et al., 1998). Par contre, au nord, la prédominance de l'espèce $S$. bovis, dont la souris blanche est considérée comme un "bon" hôte expérimental, expliquerait la bonne compatibilité vis à vis du parasite, traduite par les taux

\section{CONCLUSION ET APPLICATION DES RÉSULTATS}

Les marqueurs biologiques de compatibilité schistosomes-mollusques et de chronocercariométrie ont permis de distinguer les espèces anthropophiles de celles zoophiles du groupe Schistosoma prélevés chez l'homme et dans le milieu naturel. S. haematobium, $S$. bovis ont ainsi été retrouvés dans chacun des sites prospectés avec une prédominance de $S$. haematobium

\section{REMERCIEMENT}

Nos remerciements vont à l'endroit des autorités de l'université Félix Houphouët-Boigny qui ont accepté d'héberger et d'autoriser la réalisation des manipulations

\section{REFERENCES BIBLIOGRAPHIQUES}

Angora EK, Allienne JF, Rey O, Menan $\mathrm{H}$, Touré AO, Coulibaly JT, Raso G, Yavo W, N'Goran EK, Utzinger J, Balmer O, Boissier J, 2019. High prevalence of Schistosoma haematobium $x$ Schistosoma bovis hybrids in schoolchildren in Côte d'lvoire. Parasitology 147, 1-25. https://doi.org/10.1017/S0031182019001549.

Boissier J, Grech-Angelini S, Webster BL, Allienne JF, Huyse T, Mas-Coma S, Toulza E, Barré-Cardi H, Rollinson D, Kincaid-Smith J, 2016. Outbreak of urogenital schistosomiasis in Corsica (France): an epidemiological case study. The Lancet Infectious Diseases 16, 971979.

Cecchi P, Baldé S, Yapi YG, 2007. Mollusques hôtes intermédiaires de bilharzioses dans les petits élevés de réussite à l'infestation, le nombre et les couples de vers adultes (Vera, 1991).

En conséquence, les résultats de la chronobiologie et des infestations de souris au laboratoire suggèrent la présence de $S$. haematobium et $S$. bovis. Chez l'homme, seule l'espèce $S$. haematobium a été trouvée. Dans le milieu naturel, les deux espèces de parasites ont été trouvées dans chacun des sites prospectés avec une prédominance de $S$. haematobium au centre et $S$. bovis au nord. Des études réalisées en Côte d'Ivoire corroborent ces résultats (N'Goran,1998; Cecchi et al., 2007 ; Tian $\mathrm{Bi}$ et al., 2019). La présence des deux espèces de schistosomes au niveau des différents sites pourrait s'expliquer par l'utilisation du même point d'eau par les hommes et le bétail. La cohabitation de ces deux espèces apparentées dans les mêmes espaces géographiques, serait à la base du phénomène de l'hybridation déjà observé en Côte d'Ivoire (Angora et al., 2019 ; Tian Bi et al., 2019).

au centre et $S$. bovis au nord. Toutefois, les profils atypiques d'émergence cercarienne observés interpellent sur la présence éventuelle d'œufs de schistosomes hybrides dans les urines de l'homme. L'utilisation d'outils moléculaire pourra permettre de conforter ces observations.

au sein de leur laboratoire dans le cadre de cette étude. Nous remercions également Mr DIABATÉ Salia pour sa disponibilité et sa participation aux enquêtes.

barrages. L'eau en partage. Les petits barrages de Côte d'Ivoire 175-189.

Cheever AW, Duvall RH, Hallack TA, 1983. Hepatic fibrosis in Schistosoma haematobium-infected mice. Trans R Soc Trop Med Hyg 77, 673-679. https://doi.org/10.1016/0035-9203(83)90202

Dorsey CH, Cousin CE, Lewis FA, Stirewalt MA, 2002. Ultrastructure of the Schistosoma mansoni cercaria. Micron 33, 279-323. https://doi.org/10.1016/S0968-4328(01)000191.

Gryseels B, Polman K, Clerinx J, Kestens L, 2006. Human schistosomiasis. The Lancet 368, 1106-1118.

Huyse, T, Van den Broeck F, Hellemans B, Volckaert FAM, Polman K, 2013. Hybridisation between 
the two majors African schistosome species of humans. International journal for parasitology 43, 687-689.

Jacobs W, Van de Vijver K, Deelder A, Van Marck E, 1998. Morphometrical and immunopathological dissection of the hepatic Schistosoma haematobium granuloma in the murine host. Parasite 5, 299-306. https://doi.org/10.1051/parasite/1998054299.

Leger E, Webster JP, 2017. Hybridizations within the genus Schistosoma: implications for evolution, epidemiology and control. Parasitology 144, 65-80.

M'Bra RK, Kone B, Yapi YG, Silué KD, Sy I, Vienneau D, Soro N, Cissé G, Utzinger J, 2018. Risk factors for schistosomiasis in an urban area in northern Côte d'Ivoire. Infectious diseases of poverty 7 , 47.

Mintsa-Nguéma R, Moné $\mathrm{H}$, Ibikounlé $\mathrm{M}$, Mengué-Ngou MK, Kombila M, Mouahid G, 2014. Cercarial emergence pattern of Schistosoma haematobium from Libreville, Gabon. Parasite 21,3 . https://doi.org/10.1051/parasite/2014004.

Moné Y, Ribou AC, Cosseau C, Duval D, Théron A, Mitta G, Gourbal B, 2011. An example of molecular co-evolution: Reactive oxygen species (ROS) and ROS scavenger levels in Schistosoma mansoni/Biomphalaria glabrata interactions. International Journal for Parasitology 41, 721 730.

https://doi.org/10.1016/j.jpara.2011.01.007.

N'Goran EK, 1998. Environnement et transmission des schistosomes à œuf à éperon terminal en Côte d'Ivoire, Afrique de l'Ouest (PhD Thesis).

N'Goran EK, Utzinger J, N'Guessan AN, Müller I, Zamblé $K$, Lohourignon $K L$, Traoré $M$, Sosthène $B A$, Lengeler C, Tanner M, 2001. Reinfection with Schistosoma haematobium following schoolbased chemotherapy with praziquantel in four highly endemic villages in Côte d'Ivoire. Tropical medicine \& international health 6, 817825.

N'Guessan AN, Tian-Bi TY, Orsot NM, Yapi AK, N'Goran LL, N'Goran KE, 2015. Variabilité de la compatibilité entre Schistosoma haematobium et ses hôtes potentiels dans la zone préforestière de Côte d'Ivoire : Implications épidémiologiques. Journal of Applied Biosciences 85, 7862-7870.

OMS, 2019. Schistosomiase (bilharziose). Consulté le 1 août 2019. https://www.who.int/fr/newsroom/fact-sheets/detail/schistosomiasis.

Plouvier S, Leroy JC, Colette J, 1975. A simple method for urine filtration in the diagnosis of urinary schistosomiasis in large-scale surveys. Médecine Tropicale 35, 229-230.

Portet A, Pinaud S, Chaparro C, Galinier R, Dheilly NM, Portela J, Charriere GM, Allienne JF, Duval D, Gourbal B, 2019. Sympatric versus allopatric evolutionary contexts shape differential immune response in interaction. https://doi.org/10.1101/378034.

Tian-Bi TY, Gbocho YF, Coulibaly FH, Sangare A, N'Goran EK, 2018. Variation spatiale et temporelle de la susceptibilité de Biomphalaria pfeifferi, hôte intermédiaire de Schistosoma mansoni, Man, ouest Côte d'Ivoire. Journal of Applied Biosciences 121, 12181-1219112191. https://doi.org/10.4314/jab.v121i1.8.

Tian-Bi TY, Webster B, Konan CK, Allan F, Diakité NR, Ouattara M, Salia D, Koné A, Kakou AK, Rabone M, Coulibaly JT, Knopp S, Meïté A, Utzinger J, N'Goran EK, Rollinson D, 2019. Molecular characterization and distribution of Schistosoma cercariae collected from naturally infected bulinid snails in northern and central Côte d'Ivoire. Parasites \& Vectors 12, 117. https://doi.org/10.1186/s13071-019-3381-3.

Utzinger J, Becker SL, van Lieshout L, van Dam GJ, Knopp S, 2015. New diagnostic tools in schistosomiasis. Clinical Microbiology and Infection 21, 529-542. https://doi.org/10.1016/j.cmi.2015.03.014.

Vera C, 1991. Contribution à l'étude de la variabilité génétique des schistosomes et de leurs hôtes intermédiaires : polymorphisme de la compatibilité entre diverses populations de Schistosoma haematobium, $S$. bovis et $S$. curassoni et les bulins hôtes potentiels en Afrique de l'Ouest.

Webster BL, Alharbi MH, Kayuni S, Makaula P, Halstead F, Christiansen R, Juziwelo L, Stanton MC, LaCourse EJ, Rollinson D, 2019. Schistosome Interactions within the Schistosoma haematobium Group, Malawi. Emerging infectious diseases 25, 1245. 